\title{
Phase separation of polymer-bound particles induced by loop-mediated one dimensional effective long-range interactions
}

\author{
G. David, ${ }^{1}$ J.-C. Walter, ${ }^{1}$ C. P. Broedersz,,${ }^{2, *}$ J. Dorignac, ${ }^{1}$ F. Geniet, ${ }^{1}$ A. Parmeggiani,,${ }^{1,3}$ N.-O. Walliser, ${ }^{1}$ and J. Palmeri $\odot^{1, \dagger}$ \\ ${ }^{1}$ Laboratoire Charles Coulomb (L2C), Université de Montpellier, CNRS, Montpellier, France \\ ${ }^{2}$ Arnold Sommerfeld Center for Theoretical Physics and Center for Nanoscience, Ludwig-Maximilian-Universität München, \\ D-80333 Munich, Germany \\ ${ }^{3}$ Laboratory of Pathogen Host Interactions (LPHI), Université de Montpellier, CNRS, Montpellier, France
}

(Received 19 November 2018; revised 29 February 2020; accepted 3 August 2020; published 9 September 2020)

\begin{abstract}
The cellular cytoplasm is organized into compartments. Phase separation is a simple manner to create membraneless compartments in order to confine and localize particles like proteins. In many cases, these particles are bound to fluctuating polymers like DNA or RNA. We propose a general theoretical framework for such polymer-bound particles and derive an effective 1D lattice gas model with both nearest-neighbor and emergent long-range interactions arising from looped configurations of the fluctuating polymer. We argue that 1D phase transitions exist in such systems for both Gaussian and self-avoiding polymers and, using a variational method that goes beyond mean-field theory, we obtain the complete mean occupation-temperature phase diagram. To illustrate this model, we apply it to the biologically relevant case of ParABS, a prevalent bacterial DNA segregation system.
\end{abstract}

DOI: 10.1103/PhysRevResearch.2.033377

\section{INTRODUCTION}

The confinement of chemical species, such as RNA or proteins, within the cytoplasm is mandatory for the spatiotemporal organization of chemical activities in the cell [1]. Cells compartmentalize the intracellular space using either membrane vesicles or membraneless organelles. For the latter, cells may employ phase separation of chemical species in order to create localized high-density regions in which specific reactions may occur [2,3]. Such biological phase separation mechanisms often involve polymeric scaffolds like Ribonucleic acid (RNA) or Deoxyribonucleic acid (DNA) to bind the chemical species [4-9]. A prominent example may be the formation of localized protein-DNA complexes during bacteria DNA segregation due to the in vivo ParABS system [10-15]. Although the molecular components of this widely conserved segregation machinery have been clearly identified, their dynamical interplay and the mechanism that leads to the condensation of the complexes remain elusive. The interaction between a fluctuating polymer in a good solvent and smaller associating particles is also a general problem that goes beyond biology. There are important industrial applications that exploit the possibility of fine-tuning such systems to induce polymer-surfactant aggregation at low surfactant concentration [16].

\footnotetext{
*Present address: Department of Physics and Astronomy, Vrije Universiteit Amsterdam, 1081 HV Amsterdam, The Netherlands.

$\dagger$ john.palmeri@umontpellier.fr

Published by the American Physical Society under the terms of the Creative Commons Attribution 4.0 International license. Further distribution of this work must maintain attribution to the author(s) and the published article's title, journal citation, and DOI.
}

More generally, despite early theoretical work [16-18] and more recent extensive simulation studies [12,19-21], it is still unclear theoretically how long 1D substrates like DNA polymers interact with particles to form 3D structures essential for the cellular cycle $[4,9,22]$. Interestingly, similar organizational principles may apply to the higher order folding of chromatin and the interactions between topological domains in eukaryotic cells [22-26]. A common theme is the mechanism of protein-induced polymer loop formation via bridging interactions and the role played by these loops in structuring DNA and creating localized protein-DNA complexes. Three different basic models have been studied, mainly using simple mean-field Flory or Flory-Huggins type approaches and simulations: (i) sparse but fixed interacting sites [21,22,27,28] or block copolymers (heteropolymers) composed of fixed sequences of different monomers [24], (ii) nonattracting mobile particles that can bind simultaneously to two or more polymer sites to form bridges [19,23,29], and (iii) mobile particles that bind to a polymer and attract to form both bridging bonds [12,16-18] and possibly nearest-neighbor (NN) ones.

In the above cited studies, the focus is on the collapse of a polymer induced by polymer-particle interactions, rather than on the phase behavior of the polymer-bound particles. For example, in one interesting study [16], a simple mean field theory was used to investigate the influence of associating particles on polymer conformation. It was found that after integrating out the particle degrees of freedom the polymer could undergo partial collapse, leading to a joint self-assembly of the polymers and associating particles [16]. The complementary approach was not, however, investigated, namely the phase behavior of the polymer-bound particles once the polymer degrees of freedom have been integrated out.

It is therefore not clear from earlier studies if polymer collapse (or partial collapse) is a prerequisite for the appearance 


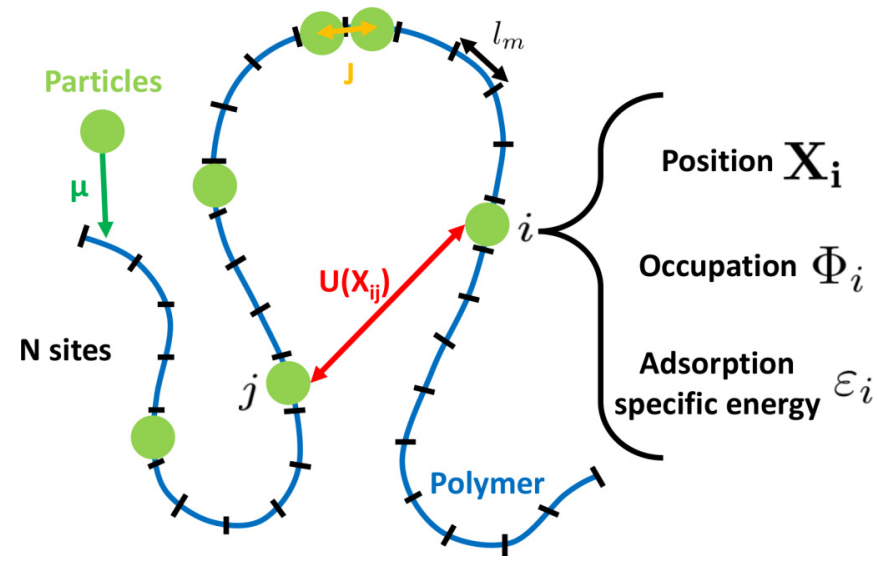

FIG. 1. Coupled polymer-particle model: The polymer in 3D is divided into $N$ monomers, each having a position vector $\mathbf{X}_{i}$, an occupation $\Phi_{i}$, and a local adsorption energy $\epsilon_{i}$. Loops form when particles far apart along the polymer interact at short range in $3 \mathrm{D}$.

of polymer-bound particle phase separation. Furthermore, previous work on case (iii) [16-18], based on mean field theory, did not address the crucial question of the range of the effective 1D long-range interactions between polymer-bound particles, necessary for determining the existence of a true phase transition.

We present here an analytical Hamiltonian approach to case (iii) by introducing a basic microscopic particle-polymer statistical mechanical model where all relevant physical parameters appear explicitly. Such a framework is needed to clarify the existence and nature of phase transitions in such systems, especially since approximate theoretical [16-18] and numerical [12] studies of finite size systems suggest phase separation-like behavior. From this model, we derive an effective 1D lattice gas model with 1D temperature-dependent long-range interactions that arise once the $3 \mathrm{D}$ conformational fluctuations of the polymer have been integrated out. We show that the existence of a phase transition in this effective model depends on the exponent describing the asymptotic power law decay of the long-range interactions. We then propose a variational method that goes beyond mean field theory (MFT) to compute the mean occupation-temperature phase diagram. We finally, for illustration, apply our model to the bacterial partition system ParABS and the formation of ParB condensates. As a result of this analysis, we propose a plausible explanation in terms of low-density phase metastability for experiments showing the existence of high-density ParB protein condensates only in the presence of specific parS binding sites.

\section{MODEL}

The polymer consists of $N$ monomers (or binding sites) with each monomer capable of accommodating one bound particle (see Fig. 1). The effective monomer length $l_{m}$ corresponds to the footprint of one particle on the polymer, measured, for example, in terms of base pairs for DNA. Each site $i$ is characterized by its position in 3D space $\mathbf{X}_{i}$, its occupation $\Phi_{i}$ (equal to 1 if a particle is bound and 0 otherwise), and its on-site binding energy $\varepsilon_{i}$ (allowing for local specific or nonspecific binding). In the particle grand- canonical ensemble, the energy of a state $\left[\Phi_{i}, \mathbf{X}_{i}\right]$ is

$$
H\left[\Phi_{i}, \mathbf{X}_{i}\right]=H_{\mathrm{P}}\left[\mathbf{X}_{i}\right]+H_{\mathrm{SRLG}}\left[\Phi_{i}\right]+H_{\mathrm{B}}\left[\Phi_{i}, \mathbf{X}_{i}\right] .
$$

The first term $H_{\mathrm{P}}\left[\mathbf{X}_{i}\right]$ describes the polymer configuration energy. The second is a $1 \mathrm{D}$ short-range lattice gas (SRLG) Hamiltonian for bound particles,

$$
H_{\mathrm{SRLG}}\left[\Phi_{i}\right]=-J \sum_{i=1}^{N-1} \Phi_{i+1} \Phi_{i}-\sum_{i=1}^{N}\left(\mu-\varepsilon_{i}\right) \Phi_{i}
$$

with NN spreading interaction coupling constant $J$ and chemical potential $\mu$. The contribution from 3D bridging interactions, giving the coupling between the bound particles and the fluctuating polymer, takes the form

$$
H_{\mathrm{B}}\left[\Phi_{i}, \mathbf{X}_{i}\right]=\frac{1}{2} \sum_{i, j}^{N} \Phi_{i} U\left(X_{i j}\right) \Phi_{j}
$$

with $X_{i j}=\left|\mathbf{X}_{i}-\mathbf{X}_{j}\right|$ and $U\left(X_{i j}\right)$ being the potential of 3D spatial interaction between particles. The prime on the sum means that $|i-j| \geqslant n_{\text {inf }}$, where $n_{\text {inf }}$ is the minimal internal distance in number of sites over which two particles can interact at long range.

The polymer conformational degrees of freedom can formally be integrated out, yielding a highly nonlinear 1D effective free energy for the bound particles including two and all higher body interactions along the chain. Given the complexity of this coupled model, we derive, using a virial (cluster) expansion [30,31], a more amenable 1D effective model that retains only short-range and two-body long-range interactions:

$$
\begin{aligned}
\frac{\mathcal{Z}}{\mathcal{Z}_{\mathrm{P}}} & =\sum_{\left\{\Phi_{i}=0,1\right\}} \exp \left[-\beta\left(H_{\mathrm{SRLG}}\left[\Phi_{i}\right]-\beta^{-1} \ln \left\langle e^{-\beta H_{\mathrm{B}}\left[\Phi_{i}, \mathbf{X}_{i}\right]}\right\rangle_{\mathrm{P}}\right)\right] \\
& \approx \sum_{\left\{\Phi_{i}=0,1\right\}} e^{-\beta \mathcal{F}_{\mathrm{LRLG}}\left[\Phi_{i}\right]}
\end{aligned}
$$

where $\beta=1 /\left(k_{\mathrm{B}} T\right),\langle\cdot\rangle_{\mathrm{P}}$ denotes an average over polymer conformations, $\mathcal{Z}_{\mathrm{P}}$ is the partition function of the bare polymer, and $\mathcal{F}_{\mathrm{LRLG}}\left[\Phi_{i}\right]$ is a $1 \mathrm{D}$ long-range lattice gas (LRLG) effective (temperature-dependent) free energy:

$$
\mathcal{F}_{\mathrm{LRLG}}\left[\Phi_{i}\right]=H_{\mathrm{SRLG}}\left[\Phi_{i}\right]-\frac{1}{2} \sum_{i, j}^{N} \Phi_{i} G_{i j} \Phi_{j}
$$

The second term of Eq. (5) is an effective 1D long-range bridging interaction between particles on the polymer that depends on the distance along the chain and arises after the chain conformational fluctuations have been integrated out, giving rise to the temperature dependence of $\mathcal{F}_{\text {LRLG }}$. The kernel,

$$
G_{i j}=4 \pi \beta^{-1} \int_{0}^{\infty} d R R^{2}\left[e^{-\beta U(R)}-1\right] P_{i j}(R),
$$

is obtained by performing a generalized virial expansion (assuming isotropy) with

$$
P_{i j}(R)=\left\langle\delta\left(R-\left|\mathbf{X}_{i}-\mathbf{X}_{j}\right|\right)\right\rangle_{\mathrm{P}},
$$

the monomer-monomer polymer probability distribution function (PDF), i.e., the probability that monomers $i$ and $j$ be 
separated by a distance $R$ in space. The effective free energy $\mathcal{F}_{\text {LRLG }}$ is therefore completely defined by the polymer and particle parameters. The effective long-range interaction encoded by the kernel $G_{i j}$ implicitly sums over all possible loops formed by the polymer segment bounded by the two bridging particles. This approach accounts exactly for twobody interactions and should therefore be valid for sufficiently low polymer monomer 3D spatial density (as in Flory-type approximations [18,32-36]). There will be no restriction, however, on the 1D occupation along the polymer.

The possibility that the LRLG model exhibit a phase separation, while the 1D SRLG model does not, is thus completely dependent on the asymptotic behavior $|i-j| \rightarrow \infty$ of the kernel $G_{i j}$. The asymptotic behavior of $P_{i j}(R)$, describing monomer-monomer close contact, is $[33,35]$

$$
P_{i j}(R) \underset{\frac{R}{R_{i j}} \rightarrow 0}{\longrightarrow} \frac{c_{0}}{R_{i j}^{3}}\left(\frac{R}{R_{i j}}\right)^{g},
$$

where $c_{0}$ is a constant and $R_{i j}=\left\langle X_{i j}^{2}\right\rangle_{\mathrm{P}}^{1 / 2}=b|i-j|^{\nu}$ is the root-mean-square monomer $i$-to- $j$ distance with $b$ as the effective Kuhn length. The exponents $v$ and $g$ depend on the chosen polymer statistics. In the absence of polymer connectivity, the monomers form an ideal gas and $P_{i j}(R)$ is replaced by the inverse system volume $V^{-1}$ in Eq. (6). The above approach then reduces to the usual nonideal gas virial expansion. To recover the Flory approach [18], the PDF is replaced by the inverse of the volume defined by the polymer radius of gyration. Such an approximation leads to an infinite-range model that leaves out crucial particle-particle correlations arising from polymer connectivity and gives results that are not consistent with those presented here (see Appendix A). In reality, bound particles closer on the chain experience enhanced two-body interactions (down to a lower limit imposed by polymer rigidity and self-avoidance).

By inserting Eq. (8) in Eq. (6), we obtain the asymptotic behavior of the long-range interaction, $G_{i j} \sim|i-j|^{-\alpha}$ with $\alpha=(3+g) \nu$. The effective 1D LRLG model clearly falls into the universality class of the well-known 1D long-range Ising model (LRIM) [37], aside from an additional NN interaction that also appears in the effective inverse square LRIM approach to the Kondo problem [38]. The exponent $\alpha$ is the key parameter to predict phase transitions in the LRIM [39]. Ferromagnetic-like phase transitions occur for a positive kernel and $1<\alpha<2$ (Dyson criterion) and critical exponents are classical for $1<\alpha<3 / 2$ [40]. The case $\alpha=2$ leads to the 1D analog of the Berezinky-Kosterlitz-Thouless phase transition $[38,41]$.

Interestingly, the Dyson criterion depends here only on the polymer properties and it is straightforward to obtain the values of $\alpha$ for the Gaussian and self-avoiding polymer (SAP) distributions. For a Gaussian polymer, $v=1 / 2$ and $g=0$, and therefore $\alpha=3 / 2$. For a SAP $\alpha \approx 1.92$, since $v \approx 0.588$ and $g \approx 0.27$ [33]. Therefore, the Dyson criterion for $\alpha$ is fulfilled and these two polymer models are expected to lead to phase separation. For an infinite compact globular polymer, we expect Gaussian behavior for interior monomers owing to internal screening of polymer self-avoidance $[42,43]$. Typical polymer conformational statistics therefore lead to a
LR interaction decay exponent $\alpha$ that ensures the existence of a $1 \mathrm{D}$ phase transition for bound particles.

\section{VARIATIONAL METHOD}

Using a variational method [31], we proceed by finding the coexistence and spinodal curves to construct the entire LRLG phase diagram in the absence of specific binding sites. To do so, we absorb the uniform nonspecific binding energy into the definition of the chemical potential and rewrite the free energy $\mathcal{F}_{\text {LRLG }}$ as the sum of two parts by introducing a variational parameter $\mu_{0}$ :

$$
\mathcal{F}_{\text {LRLG }}\left[\Phi_{i}\right]=H_{0}+\Delta H
$$

where

$$
H_{0}=-J \sum_{i=1}^{N-1} \Phi_{i+1} \Phi_{i}-\mu_{0} \sum_{i=1}^{N} \Phi_{i}
$$

and

$$
\Delta H=-\frac{1}{2} \sum_{i, j}^{N} \Phi_{i} G_{i j} \Phi_{j}-\left(\mu-\mu_{0}\right) \sum_{i=1}^{N} \Phi_{i} .
$$

$H_{0}$ is just the Hamiltonian of another 1D SRLG [see Eq. (2)] with an effective chemical potential $\mu_{0}$ and therefore has the advantage of being exactly solvable. For $J=0$, the variational method is equivalent to the MFT one, which consists in moving the $\mathrm{NN}$ interaction (term in $J$ ) from $H_{0}$ to $\Delta H$ (see Appendix B). MFT, which incorrectly predicts a 1D phase in the absence of bridging, is improved by the optimal choice for $\mu_{0}$ when $J>0$, because correlation effects, missed entirely by MFT, are approximately accounted for in the variational $H_{0}$. This variational method is exact for the (unphysical) infinite-range lattice gas (or Ising model $[44,45]$ ) (for which $G_{i j}$ is independent of $i-j$ and inversely proportional to $N$ ), and therefore we expect it to lead to reasonably accurate results for the LRLG.

The division in Eq. (9) leads to a trial grand potential

$$
\Omega_{\mathrm{V}}=\Omega_{0}+\langle\Delta H\rangle_{0} \geqslant \Omega_{\mathrm{LRLG}},
$$

where $\Omega_{0}$ is the grand potential related to $H_{0}$ and $\langle\cdot\rangle_{0}$ denotes an average with respect to $H_{0}$. In the thermodynamic limit $(N \rightarrow \infty), \Omega_{0}=-N k_{\mathrm{B}} T \ln \lambda_{+}$, where $\lambda_{+}$is the largest of the two eigenvalues $\lambda_{ \pm}$which arise from the transfer matrix method applied to the SRLG model [46]:

$$
\lambda_{ \pm}=e^{Y}\left[\cosh (Y) \pm \sqrt{\sinh ^{2}(Y)+e^{-\beta J}}\right]
$$

with $Y=\beta\left(J+\mu_{0}\right) / 2$. The second term in $\Omega_{\mathrm{V}}$,

$$
\langle\Delta H\rangle_{0}=\frac{1}{2} \sum_{i, j}^{N}{ }^{\prime} G_{i j}\left\langle\Phi_{i} \Phi_{j}\right\rangle_{0}-\left(\mu-\mu_{0}\right) \sum_{i=1}^{N}\left\langle\Phi_{i}\right\rangle_{0},
$$

involves the mean occupation in the ensemble $H_{0}, \Phi_{0} \equiv$ $\left\langle\Phi_{i}\right\rangle_{0}$, where

$$
\left\langle\Phi_{i}\right\rangle_{0}=-\frac{1}{N} \frac{\partial \Omega_{0}}{\partial \mu_{0}}=\frac{1}{2}\left(1+\frac{\sinh (Y)}{\sqrt{\sinh ^{2}(Y)+e^{-\beta J}}}\right),
$$

and the two-site correlation function,

$$
\left\langle\Phi_{i} \Phi_{j}\right\rangle_{0}=\Phi_{0}^{2}+\Phi_{0}\left(1-\Phi_{0}\right) e^{-|i-j| / \xi_{\mathrm{LG}}},
$$


in the thermodynamic limit with $\xi_{\mathrm{LG}}=-1 / \ln r_{\mathrm{LG}}$ being the SRLG correlation length and $r_{\mathrm{LG}} \equiv \lambda_{-} / \lambda_{+}$. The optimization equation

$$
\left(\partial \Omega_{\mathrm{V}} / \partial \mu_{0}\right)_{\mu_{0}=\mu_{0}^{*}}=0
$$

gives the optimal value $\mu_{0}^{\star}$ of $\mu_{0}$ :

$$
\begin{aligned}
\mu-\mu_{0}^{\star}= & 2 \Phi_{0}^{\star}\left[S^{\prime}-S\right]-S^{\prime} \\
& -\Phi_{0}^{\star}\left(1-\Phi_{0}^{\star}\right)\left(1-2 \Phi_{0}^{\star}\right) S^{\prime \prime} \beta\left(\frac{\partial \Phi_{0}}{\partial \mu_{0}}\right)_{\mu_{0}=\mu_{0}^{\star}}^{-1}
\end{aligned}
$$

with $\Phi_{0}^{\star}=\Phi_{0}\left(\mu_{0}^{\star}\right)$ and where the sums $S, S^{\prime}$, and $S^{\prime \prime}$, defined as

$$
\begin{gathered}
S=\sum_{k=n_{\text {inf }}}^{\infty} G_{k}, \\
S^{\prime}=\sum_{k=n_{\text {inf }}}^{\infty} G_{k} r_{\mathrm{LG}}^{k}, \\
S^{\prime \prime}=\sum_{k=n_{\text {inf }}}^{\infty} G_{k} k r_{\mathrm{LG}}^{k}
\end{gathered}
$$

depend crucially on the long-range behavior of the kernel $G_{i j}=G_{i-j}$ (the equality, arising from translational invariance, holds in the thermodynamic limit) (see Appendix C).

The best variational approximation to the exact grand potential $\Omega_{\mathrm{LRLG}}$ is the optimal grand potential, $\Omega_{\mathrm{V}}^{\star}=\Omega_{\mathrm{V}}\left(\mu_{0}^{\star}\right)$, from which we obtain the average site occupation $\Phi \equiv$ $-N^{-1} \partial \Omega_{\mathrm{V}}^{\star} / \partial \mu$. This last definition, along with the optimization condition, leads to $\Phi=\Phi_{0}^{\star}$ and since Eq. (15) can be inverted to obtain $\mu_{0}^{\star}$ in terms of $\Phi_{0}^{\star}$, it is possible to write $\Omega_{\mathrm{V}}^{\star}$ entirely in terms of $\Phi$ :

$$
\begin{aligned}
\frac{\Omega_{\mathrm{V}}^{\star}}{N}= & \frac{\Omega_{0}(\Phi)}{N}+\Phi^{2}\left(S-S^{\prime}\right) \\
& +\Phi^{2}(1-\Phi)(1-2 \Phi) \beta S^{\prime \prime}\left(\frac{\partial \Phi_{0}}{\partial \mu_{0}}\right)_{\mu_{0}=\mu_{0}^{\star}}^{-1}
\end{aligned}
$$

The quantities $\lambda_{+}, r_{\mathrm{LG}}$, and $\partial \Phi_{0} / \partial \mu_{0}$ are functions of $\mu_{0}^{\star}$, which can be written explicitly, by inverting Eq. (15), as a function of $\Phi$ :

$$
\beta \mu_{0}^{\star}=2 \ln \left[A \sqrt{B}+\sqrt{1+A^{2}(B-1)}\right]-\ln \left(1-A^{2}\right)-\beta J,
$$

where $A \equiv 2 \Delta \Phi$ with $\Delta \Phi=\Phi-\Phi_{\mathrm{c}}$ the distance from the critical occupation $\left(\Phi_{\mathrm{c}}=1 / 2\right.$ by particle-hole symmetry) and $B \equiv e^{-\beta J}$. For instance, $\beta \Omega_{0}(\Phi) / N$ can be written explicitly as a function of $\Phi$,

$$
\begin{aligned}
\frac{\beta \Omega_{0}(\Phi)}{N}= & \ln \left(1-A^{2}\right)-\ln \left(\sqrt{1+A^{2}(B-1)}+A \sqrt{B}\right) \\
& -\ln \left(\sqrt{1+A^{2}(B-1)}+\sqrt{B}\right),
\end{aligned}
$$

and the derivative appearing in Eq. (20) can be written as

$$
\left(\frac{\partial \Phi_{0}}{\partial \mu_{0}}\right)_{\mu_{0}=\mu_{0}^{\star}}=\frac{\beta e^{-\beta J} \cosh \left[\frac{\beta}{2}\left(\mu_{0}^{\star}+J\right)\right]}{\left\{\sinh ^{2}\left[\frac{\beta}{2}\left(\mu_{0}^{\star}+J\right)\right]+e^{-\beta J}\right\}^{\frac{3}{2}}},
$$

which when combined with Eq. (21) leads to an explicit function of $\Phi$.

With the above analytical variational expressions for the chemical potential $\mu$ and the LRLG pressure $P \approx$ $-\Omega_{\mathrm{V}}^{\star} /\left(N l_{m}\right)$ as functions of $\Phi$, we can obtain the coexistence and spinodal curves $[47,48]$. The coexistence curve is defined by the equality of $\mu$ and $P$ in the phases of high $\left(\Phi_{h}\right)$ and low $\left(\Phi_{l}\right)$ occupation,

$$
\mu\left(\Phi_{l}\right)=\mu\left(\Phi_{h}\right)
$$

and

$$
P\left(\Phi_{l}\right)=P\left(\Phi_{h}\right),
$$

and the critical point $\left(\Phi_{\mathrm{c}}, T_{\mathrm{c}}\right)$ by

$$
\partial P / \partial \Phi=\partial^{2} P / \partial \Phi^{2}=0 .
$$

Owing to particle-hole symmetry, $\Phi_{h}+\Phi_{l}=1$ and $\Phi_{\mathrm{c}}=$ $1 / 2$, the full coexistence curve, $T_{\text {coex }}(\Phi)$, can be obtained by solving a single equation, such as

$$
\mu\left(\Phi_{l}\right)=\mu\left(1-\Phi_{l}\right)
$$

or

$$
P\left(\Phi_{l}\right)=P\left(1-\Phi_{l}\right) .
$$

The spinodal curve, $T_{\mathrm{sp}}(\Phi)$, which fixes the limits of metastability, is defined by the divergence of the isothermal compressibility, or

$$
\partial P / \partial \Phi=0 .
$$

The critical temperature is found in the limit $\Phi \rightarrow \Phi_{\mathrm{c}}=$ $1 / 2$. This leads to the variational critical temperature as a solution to the following implicit equation:

$$
\frac{T_{\mathrm{c}}^{\mathrm{V}}}{T_{\mathrm{r}}}=\frac{1}{2 k_{B} T_{\mathrm{r}}}\left[\left(S_{\mathrm{c}}-S_{\mathrm{c}}^{\prime}\right) \exp \left(\frac{J}{2 k_{B} T_{\mathrm{c}}^{\mathrm{v}}}\right)-S_{\mathrm{c}}^{\prime \prime}\right],
$$

where the subscript $\mathrm{c}$ indicates quantities evaluated at the critical point and $T_{r}=300 \mathrm{~K}$ is the room temperature.

For simplicity, we illustrate our results for the case of an attractive square-well (SW) particle interaction of depth $u_{0}$, range $a$, and hard core $\sigma$ [21,22]:

$$
U(R)= \begin{cases}+\infty, & \text { if } R<\sigma \\ -u_{0}, & \text { if } \sigma<R<a, \\ 0, & \text { if } R>a\end{cases}
$$

where $u_{0}>0$ controls the amplitude of the attractive spatial interaction. The asymptotic long-distance behavior (for $\left.R_{i j} / b \gg 1\right)$ is therefore given by

$$
G_{i j} \underset{|i-j| \rightarrow \infty}{\longrightarrow} K_{\mathrm{SW}}|i-j|^{-\alpha},
$$

where

$$
\begin{aligned}
K_{\mathrm{SW}}= & 4 \pi \beta^{-1} \frac{c_{0}}{3+g}\left(\frac{\sigma}{b}\right)^{3+g} \\
& \times\left\{\left(e^{\beta u_{0}}-1\right)\left[\left(\frac{a}{\sigma}\right)^{3+g}-1\right]-1\right\} .
\end{aligned}
$$

This model allows us to illustrate generic behavior for potentials with short-range repulsion and longer range attraction: $K_{\mathrm{SW}}$ is positive (attractive) at sufficiently low $T$ and decreases 

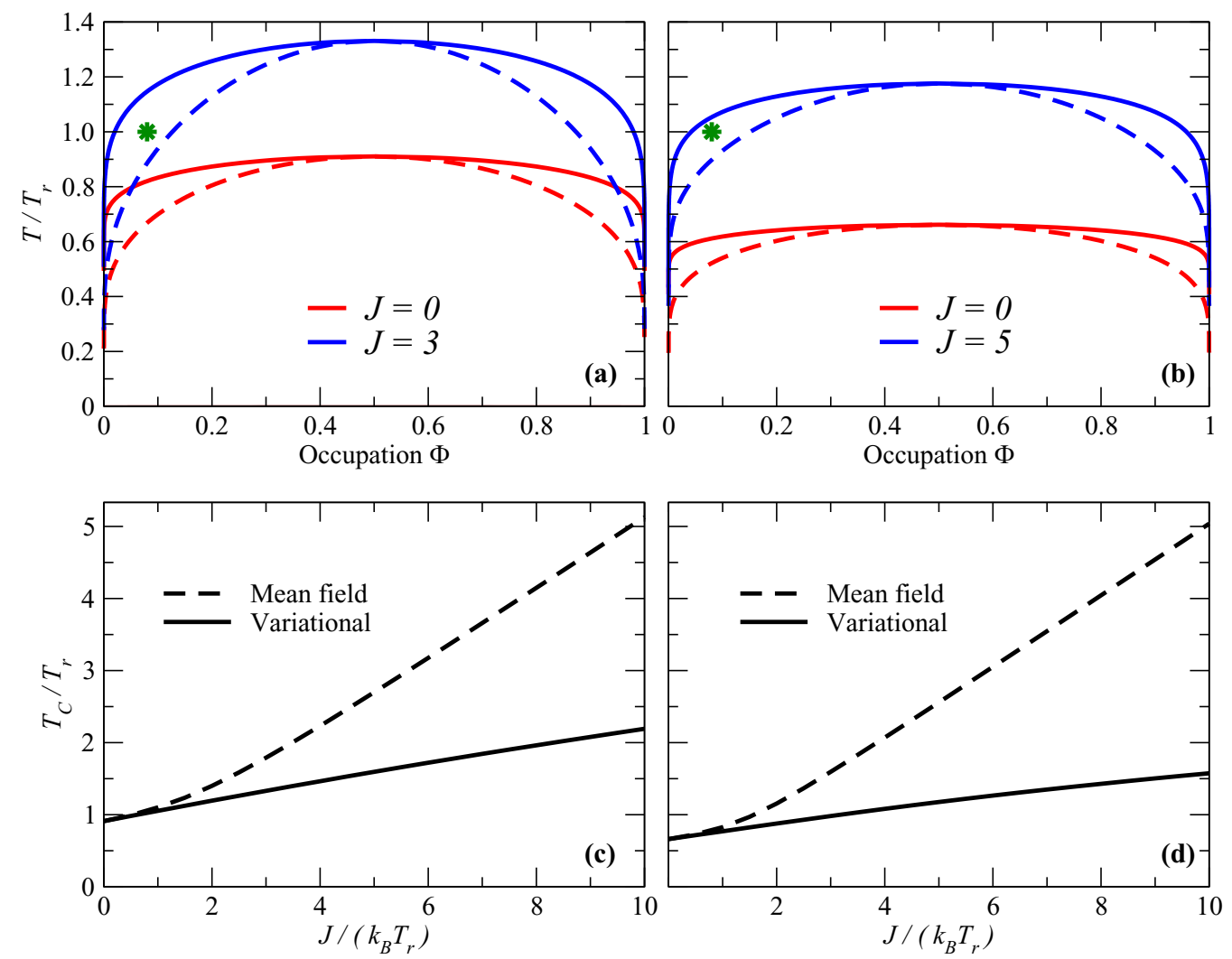

FIG. 2. Phase diagrams for polymer-bound particles. Model parameters (see text): $l_{m}=5.44 \mathrm{~nm}\left(16 \mathrm{bp}\right.$ footprint), $\sigma=l_{m}, b=\sqrt{2 l_{m} l_{p}}=$ $23.6 \mathrm{~nm}, n_{\text {inf }}=10, a=2 \sigma$, and $u_{0}=3 k_{\mathrm{B}} T_{\mathrm{r}}\left(l_{p}=51 \mathrm{~nm}\right.$ is the persistence length). Green star, biological conditions for the bacterial F-plasmid ( $\Phi=0.08$ at room temperature $T_{\mathrm{r}}$ ). (a) Gaussian polymer. Solid (dotted) line represents the coexistence (spinodal) curve for $J=0$ (red) and $J=3 k_{\mathrm{B}} T_{\mathrm{r}}$ (blue). (b) Self-avoiding polymer (SAP) with $J=0$ (red) and $J=5 k_{\mathrm{B}} T_{\mathrm{r}}$ (blue). (c) Critical temperature $T_{\mathrm{c}}$ for the Gaussian polymer: variational approach (solid line) and MFT (dotted line). (d) Same as panel (c), but for the SAP.

monotonically with decreasing slope for increasing temperature, eventually becoming negative (repulsive) at sufficiently high $T$ due to short-range repulsion. In the attractive regime of interest here, $K_{\mathrm{SW}}$ increases with $u_{0}$ and $a$ and decreases with the Kuhn length $b, \sigma$, and polymer exponent $g$ because chain stiffness and polymer self-avoidance inhibit particle-particle bridging.

\section{PHASE SEPARATION IN THE PARABS PARTITION SYSTEM}

We apply our LRLG model with an appropriately parameterized SW potential to investigate the possible role of phase separation in the $\operatorname{ParABS}$ partition system. This system ensures the segregation of autonomous DNA strands, such as the F-plasmid in E. coli [49], but also the origin domain of chromosomes in most bacteria [50]. This molecular machinery is composed of three components: a DNA sequence parS and two protein species ParB and ParA. We focus on one of its key elements, namely the formation of ParB clusters. ParB proteins can bind to DNA nonspecifically and specifically on the parS sequence [51]. Once bound to DNA, ParB proteins can mutually interact, leading to the formation of ParB $S$ partition complexes $[12,49]$. Although we now have a better understanding of segregation dynamics [13], the conditions of complex formation are still poorly understood. Experiments
$[10,11]$ show that without the parS sequence, bacteria present a homogeneous ParB distribution in the cell, while with parS a ParBS complex forms.

Our goal is to investigate whether the formation of ParBS complexes could be the result of a 1D phase separation between states of high and low ParB occupation on the DNA nucleated by $p a r S$, qualitatively similar to conventional liquidvapor phase separation in metastable situations. To reach this goal, we establish the equilibrium phase diagram in the absence of parS and locate the position of the biological system (assuming that active processes are only important in the segregation of already formed ParBS complexes [13]).

The available data for ParB allow us to parametrize the LRLG model at room temperature $T_{\mathrm{r}}=300 \mathrm{~K}$ (see Fig. 2): The truncated F-plasmid studied experimentally is a short circular DNA strand of linear size $60 \mathrm{kbp}$. There are on average $\approx 300$ ParB present on the DNA (each with a $l_{m}=16 \mathrm{bp}$ footprint) [52] and therefore $60000 / 16=3750$ possible nonspecific ParB binding sites, leading to a mean occupation $\Phi \approx 300 / 3750=0.08$.

We choose the hard core diameter to be equal to the ParB footprint, $\sigma=l_{m} \approx 5.44 \mathrm{~nm}$. From the known persistence length of DNA, $l_{p}=51.0 \mathrm{~nm}$, the Kuhn length $b=\sqrt{2 l_{p} l_{m}}$ $[33,43]$ is equal to $23.6 \mathrm{~nm}$, and the lower cutoff $n_{\text {inf }}=$ $\left\lceil 1_{p} / l_{m}\right\rceil=10$ (loops shorter than $l_{p}$ are sharply repressed by bending rigidity $[9,20])$. To complete the parametrization of 


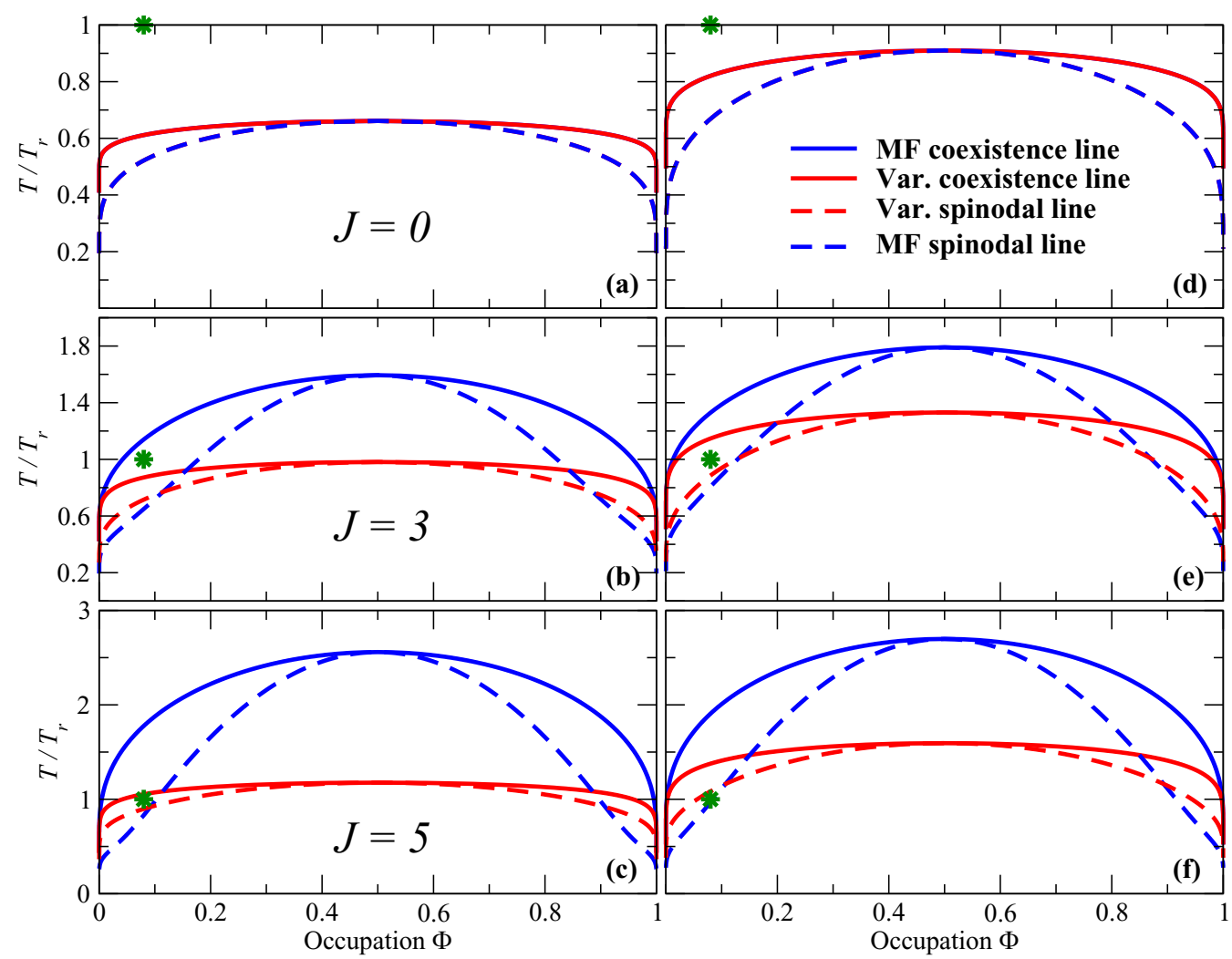

FIG. 3. [(a)-(c)] Results for a SAP. [(d)-(f)] Results for a Gaussian polymer. First line, $J=0$. Second line, $J=3 k_{B} T_{r}$. Third line, $J=$ $5 k_{B} T_{r}$ (the variational and mean field approaches give the same results for $J=0$ ). The model parameters are those used in Fig. 2.

the LRLG model, we make the following reasonable choices for the range and depth of the attractive part of the SW potential, $a=2 \sigma$ and $u_{0}=3 k_{\mathrm{B}} T_{\mathrm{r}}$, respectively. With this choice of parameters, the LRLG kernel remains positive (attractive) up to a very high temperature (more than 20 times $T_{\mathrm{r}}$ for both polymer models studied here).

We have checked that using the asymptotic form of the kernel

$$
K_{i j}=K_{\mathrm{SW}}|i-j|^{-\alpha}
$$

in place of the full one, $G_{i j}$, leads to accurate results (see Appendix C). The coefficient $c_{0}$ is equal to $[3 /(2 \pi)]^{3 / 2}$ for the Gaussian polymer (see Appendix C) and 0.58 for the SAP (estimated from exact enumeration data for open and closed chains of length $N=22$ [53]). For this choice of parameters $\beta K_{\mathrm{SW}}$ at $T_{\mathrm{r}}$ is equal to 2.26 and 3.03 for a Gaussian polymer and SAP, respectively (the higher value for the SAP arises from a higher SAP value for $c_{0}$, which compensates for the opposing effect of a larger $g$ ).

Figures 2(a) and 2(b) show the phase diagrams obtained using Gaussian polymer or SAP statistics [54]. We observe that the critical temperature $T_{\mathrm{c}}^{\mathrm{V}}$, which is the solution to Eq. (30), grows with $J$ [Figs. 2(c) and 2(d)] and that this effect is severely overestimated by MFT, for which (see Appendix D)

$$
\frac{T_{\mathrm{c}}^{\mathrm{MFT}}}{T_{\mathrm{r}}}=\frac{1}{2 k_{\mathrm{B}} T_{\mathrm{r}}}\left[J+S\left(T_{\mathrm{c}}^{\mathrm{MFT}}\right)\right] .
$$

In the asymptotic kernel approximation adopted here (see Appendix C)

$$
S(T)=\sum_{k=n_{\mathrm{inf}}}^{\infty} G_{k} \approx K_{\mathrm{SW}}(T)\left[\zeta(\alpha)-\sum_{k=1}^{n_{\mathrm{inf}}-1} \frac{1}{k^{\alpha}}\right]
$$

with $\zeta$ being the Riemann zeta function.

A simple approximation based on the weak temperature dependence of $K_{\mathrm{SW}}(T)$ for $T>T_{\mathrm{r}}$ and obtained by evaluating $S$ in Eq. (35) at $T_{\mathrm{r}}$ explains the linear dependence of $T_{\mathrm{c}}^{\mathrm{MFT}}$ on $J$ for large $J$ (see Appendix D). The temperature dependence of the kernel is, however, crucial in determining the critical temperature for small $J$. The variational result for the critical temperature is also close to being linear in $J$ for large $J$ and heuristically can be obtained from MFT by evaluating $S$ at $T_{\mathrm{r}}$ and replacing $J$ by $J / 3$.

The expression (36) indicates how the critical temperature is crucially determined by $n_{\text {inf }}$, the polymer persistence length in site number, by reducing the weight of the LR interaction contribution $[9,20]$. The relatively large value of $n_{\text {inf }}=10$ implies that the coefficient of the $K_{\mathrm{SW}}$ term in Eq. (36) is reduced by $75 \%$ for the Gaussian polymer and $92 \%$ for the SAP (with respect to $n_{\text {inf }}=0$ ). In Fig. 2 , the lower $T_{\mathrm{c}}$ shown by the SAP compared with the Gaussian polymer at constant $J$ is due to the faster decay of the LR interaction (larger $\alpha$ ), despite the larger value of the SAP $K_{\mathrm{SW}}$ (see Appendix C). The critical temperature $T_{\mathrm{c}}$ is nonzero even for $J=0$, but is far below room temperature. Therefore, the system does not exhibit phase separation without spreading interactions at this temperature. Both short-range spreading with reasonable 
biological values for $J\left(\approx 3-6 k_{\mathrm{B}} T_{\mathrm{r}}\right)$ and long-range bridging interactions are thus required at room temperature to form ParB condensates in our model, as suggested by Monte Carlo simulations [12] and experiments [49,55].

We show in Fig. 3 supplementary phase diagrams for Gaussian and self-avoiding polymers (SAPs) and various values of $J$ to provide examples of how mean field phase diagrams deviate substantially from the variational ones as the $\mathrm{NN}$ interaction $J$ increases. These results also illustrate the global dependence of the phase diagrams on this key model parameter.

The position of this system at room temperature in the occupation-temperature phase diagram [green star in Figs. 2(a) and 2(b)] shows that for reasonable biological values of $J$ the system without parS exists in the low occupation metastable coexistence region, providing a plausible explanation for the experimental observations [11,12]. Thus, specific ParB binding to parS could provide the energy required to overcome the nucleation barrier and allow the system to switch from the metastable low occupation homogeneous state to the stable coexistence phase, in which ParB proteins form a stable high occupation (liquid) cluster on the DNA around parS, surrounded by a low ParB density (vapor) background. Experimentally, this system should follow the conventional behavior of liquid-vapor phase transitions:

(1) In the low occupation metastable region, the system can form relatively high-density ParB $S$ complexes with only a small total number of intracellular proteins.

(2) ParB over- or underexpression will favor or repress the formation of ParBS complexes depending on the position in the phase diagram. Indeed, systems without parS but with sufficiently high ParB occupation would be in the unstable coexistence area and should therefore form protein (liquid) droplets spontaneously in a low occupation (vapor) background, the homogeneous state being unstable in this case.

(3) In contrast, systems with too few ParB proteins would be in the low occupation vapor region, losing the ability to form complexes even in the presence of parS.

Experimental evidence for such global trends may already exist in in vitro single-molecule experiments $[55,56]$.

\section{SUMMARY AND CONCLUSIONS}

We have proposed a general theoretical framework for the physics of particles interacting on a polymer fluctuating in $3 \mathrm{D}$ that leads naturally to an effective 1D LRLG model. We established a criterion for the existence of a 1D phase transition based on the exponent $\alpha$ controlling the asymptotic decay of the LR interactions, which depends only on the polymer exponents $v$ and $g$. Since this criterion is satisfied for standard polymer models, the conformational fluctuations of linear structures like DNA produce effective 1D longrange interactions between bound particles that lead to $1 \mathrm{D}$ particle phase separation along the polymer. We used our theoretical approach to construct the whole phase diagram of the ParB proteins which are part of a prevalent bacterial DNA segregation system and concluded that the formation of ParB condensates could plausibly result from parS nucleated phase separation in the low ParB occupation metastable region. This general mechanism for triggering the formation of polymer-bound protein complexes via small nucleation sites may generally play an important role in membraneless cell compartmentalization and in industrially important polymersurfactant systems [16].

The phase diagrams for the same model, but without nearest neighbor interactions, presented in Figs. 5 and 6 of Ref. [18] were obtained using a mean field Flory approach that predicts phase separation only in the collapsed globule state but not in the swollen and ideal polymer states. These phase diagrams cannot be used to explain the formation of ParB condensates, because they show phase coexistence only in the very high occupation regime (greater than $75 \%$ coverage on both branches of the phase coexistence curves). Contrary to our results, these results cannot therefore explain the low occupancy background phase needed to explain the experimental ParABS results. There is also evidence coming from simulations against the ParB condensates being in a compact globule state [12].

Our method may also be used to derive the 1D particle distribution along the polymer and the 3D particle density of the condensate that forms around a specific binding site, both of which are accessible experimentally [11,49]. It could also be generalized to treat models (i) and (ii) evoked in the introduction (see Appendix E). Finally, to facilitate quantitative testing of the present approach, it would also be of great interest to apply it to the analysis of industrially important polymer-surfactant systems [16], as well as to pursue experimental and theoretical studies of in vitro biomimetic systems $[55,56]$.

\section{ACKNOWLEDGMENTS}

This project received partial financial support from the French Agence Nationale de la Recherche [Imaging and Modeling Bacterial Mitosis (IBM) Project No. ANR-14CE09-0025-01], the CNRS Défi Inphyniti (Projet Structurant No. 2015-2016), and the LabEx NUMEV (ANR-10LABX-0020) within the I-SITE MUSE of the Universite de Montpellier [No. AAP 2013-2-005, No. 2015-2-055, No. 2016-1-024, and Flagship Project Gene Expression Modeling (2017-2020)] (G.D., J.C.W., J.D., F.G., A.P., N.O.W., J.P.). G.D. acknowledges doctoral thesis and A.T.E.R postdoctoral support from the French Ministère de l'Enseignement supérieur, de la Recherche et de l'Innovation. This work was supported in part by a Modélisation pour le Vivant French CNRS Grant (CoilChrom project). This project was also supported by the Deutsche Forschungsgemeinschaft (DFG) Grant No. TRR174 (C.P.B.). The authors would like to thank J.-Y. Bouet, M. Nollmann, and N. Wingreen for interesting discussions on the ParABS system.

\section{APPENDIX A: FLORY APPROACH}

We present here a critical analysis of the early work by Dormidontova et al. [18] and show by comparison with our own work that the Flory-type approach they adopt, usually a natural starting point to tackle difficult polymer problems, is inadequate for understanding the phase behavior of the system studied. It is important to understand why a Flory-type approach [18,32-36] fails in this case. 
The work by Dormidontova et al. examines the same general problem that we do, that of interacting particles bound to a fluctuating polymer, but as a starting point they immediately formulate the problem in the form of a Flory mean field theory. This type of approach, despite its interest, allows neither the model to be put on a solid basis, nor, because of its ad hoc nature, the physics of the system to be studied in a coherent statistical mechanical framework.

We start by sketching a method for obtaining the Flory approach in the current context. We underline that this is not a derivation of the Flory approach because it relies on an unjustified step that has no rigorous foundations.

The partition function for a polymer of length $N$ and effective monomer length $b$

$$
\mathcal{Z}_{\mathrm{P}}=4 \pi b^{-3} \int_{0}^{\infty} d R R^{2} Q_{\mathrm{P}}(R),
$$

can be written in terms of the restricted polymer partition function, $Q_{\mathrm{P}}(R)$ (with end-to-end distance constrained to be $R)$. The (normalized) probability distribution function, $P_{\mathrm{ee}}(R)$, which is related to $Q_{\mathrm{P}}(R)$ by $P_{\mathrm{ee}}(R)=b^{-3} Q_{\mathrm{P}}(R) / \mathcal{Z}_{\mathrm{P}}$, gives the probability to find the polymer in a state with an end-toend distance equal to $R$. The mean-square end-to-end distance is

$$
R_{\mathrm{ee}}^{2}=\left\langle R^{2}\right\rangle=4 \pi \int_{0}^{\infty} d R R^{4} P_{\mathrm{ee}}(R)
$$

Since we expect $P_{\text {ee }}$ to be a function of $R$ only in the combination $R / R_{\mathrm{ee}}$, we introduce the scaling function $p_{\mathrm{ee}}$, via

$$
P_{\mathrm{ee}}(R)=R_{\mathrm{ee}}^{-3} p_{\mathrm{ee}}\left(R / R_{\mathrm{ee}}\right),
$$

which allows us to rewrite $\mathcal{Z}_{\mathrm{P}}$ as

$$
\mathcal{Z}_{\mathrm{P}} \sim \int_{0}^{\infty} d x \exp [-\Gamma(x)]
$$

using the change of variables $x=R / R_{\mathrm{ee}}$ and introducing an effective end-to-end free energy,

$$
\Gamma(x)=-\ln \left[p_{\mathrm{ee}}(x)\right]-2 \ln (x),
$$

that fixes the weight of a configuration with an end-toend distance $R=x R_{\mathrm{ee}}$ in the full partition function. The Flory approach can be couched in the form of a saddlepoint approximation, given by $\Gamma^{\prime}(x)=0$, to obtain an approximation for the end-to-end distance and full partition function.

Given the known form of $P_{\mathrm{ee}}$ for a Gaussian polymer,

$$
P_{\mathrm{G}}(R)=\left(\frac{3}{2 \pi R_{\mathrm{G}}^{2}}\right)^{3 / 2} \exp \left[-\frac{3}{2}\left(\frac{R}{R_{\mathrm{G}}}\right)^{2}\right],
$$

where the Gaussian polymer end-to-end distance is $R_{\mathrm{G}}=$ $b N^{1 / 2}$ ( $b$ is the Kuhn length), it is straightforward in this case to find the Gaussian polymer scaling function

$$
p_{\mathrm{G}}(\alpha)=\left(\frac{3}{2 \pi}\right)^{3 / 2} \exp \left[-\frac{3}{2} \alpha^{2}\right]
$$

and the effective free energy, $\Gamma_{\mathrm{G}}$, as a function of the so-called swelling factor $\alpha=R / R_{\mathrm{G}}$,

$$
\Gamma_{\mathrm{G}}(\alpha)=-2 \ln \alpha+\frac{3}{2} \alpha^{2}-\frac{3}{2} \ln \left(\frac{3}{2 \pi}\right) .
$$

Using the Flory approach, $\Gamma_{\mathrm{G}}^{\prime}(\alpha)=0$, we can obtain an approximation, $R_{\mathrm{G}}^{\mathrm{SP}}$, for the end-to-end distance and full partition function for the simple Gaussian polymer: We recover the exact end-to-end distance scaling with less than $20 \%$ error for the ratio $R_{\mathrm{G}}^{\mathrm{SP}} / R_{\mathrm{G}}$.

To take into account monomer-monomer $(\mathrm{mm})$ and bound particle-bound particle (pp) interactions, we can now proceed as we did for the full partition function (see main text) and perform a generalized virial expansion, but with the extra complication that we are now working with the constraint that the polymer end-to-end distance be fixed at $R=\alpha R_{\mathrm{G}}$. We are also now treating $\mathrm{mm}$ and pp interactions on the same footing, instead of treating the polymer (formally) exactly and therefore a coupling between the fluctuating polymer and bound particles only appears when the bare $\mathrm{mm}$ interaction, $u_{\mathrm{mm}}(r)$, is different from the $\mathrm{pp}$ interaction one, $u_{\mathrm{pp}}(r)$ :

$$
\begin{aligned}
\Gamma\left[\alpha ; \Phi_{i}\right] \approx & -2 \ln \alpha+\frac{3}{2} \alpha^{2}-\frac{3}{2} \ln \left(\frac{3}{2 \pi}\right) \\
& -\frac{1}{2} \sum_{i, j}^{N} \Phi_{i}\left[G_{i j}^{\mathrm{pp}}(R)-G_{i j}^{\mathrm{mm}}(R)\right] \Phi_{j} \\
& -\frac{1}{2} \sum_{i, j}^{N} G_{i j}^{\mathrm{mm}}+H_{\mathrm{SRLG}}\left[\Phi_{i}\right],
\end{aligned}
$$

where both the constrained kernel for monomer-monomer interactions,

$$
G_{i j}^{\mathrm{mm}}(R)=4 \pi b^{3} \beta^{-1} \int_{0}^{\infty} d r r^{2}\left[e^{-\beta u_{\mathrm{mm}}(r)}-1\right] P_{i j}(r ; R),
$$

and the constrained kernel for bound particle-bound particle interactions,

$$
G_{i j}^{\mathrm{pp}}(R)=4 \pi b^{3} \beta^{-1} \int_{0}^{\infty} d r r^{2}\left[e^{-\beta u_{\mathrm{pp}}(r)}-1\right] P_{i j}(r ; R),
$$

depend on the monomer-monomer $(\mathrm{mm})$ polymer constrained probability distribution function (cPDF),

$$
P_{i j}(r ; R)=\left\langle\delta\left(r-\left|\mathbf{X}_{i}-\mathbf{X}_{j}\right|\right) \delta\left(R-\left|\mathbf{X}_{1}-\mathbf{X}_{N}\right|\right)\right\rangle_{\mathrm{GP}},
$$

which describes the probability that monomers $i$ and $j$ be separated by a distance $r$ in space given that the end monomers are separated by a distance $R$ (GP denotes that the average is taken for the Gaussian polymer). It is clear that the particle-particle interaction introduced in the main text, $U(r)$, is an effective one that implicity accounts for the difference between the bare monomer-monomer two-body interaction and the bound particle-bound particle one:

$$
e^{-\beta U(r)}=1+e^{-\beta u_{\mathrm{pp}}(r)}-e^{-\beta u_{\mathrm{mm}}(r)} .
$$

(For simplicity, and following Ref. [18], we assume that the monomer-bound particle interaction is purely repulsive and identical to the monomer-monomer one.) We expect that if the $\mathrm{mm}$ interaction is approximated by a hard-core repulsion with a range that is much less than the hard core repulsion of the pp interaction, then the amplitude of the kernel (33) will not be substantially modified. 
The mean field Flory approach adopted in Ref. [18] consists in making without justification the replacement of $b^{3} P_{i j}(r ; R)$ by an effective interaction volume $R^{-3}=$ $\left(\alpha R_{\mathrm{G}}\right)^{-3}$. This replacement neglects all correlations along the polymer and leads effectively to an infinite-range model. We then recover the approach of Ref. [18] if the nearest-neighbor interaction $J$ is set to zero in the SRLG Hamiltonian, the lattice gas is treated in the mean field approximation to find the Helmoltz free energy (in the canonical ensemble), and a simple form for the third virial coefficient (independent of the particle occupation) is added in by hand.

The monomer-monomer and bound particle-bound particle second virial coefficents are given by, respectively,

$$
B_{\mathrm{mm}}=-2 \pi \int_{0}^{\infty} d r r^{2}\left[e^{-\beta u_{\mathrm{mm}}(r)}-1\right]
$$

and

$$
B_{\mathrm{pp}}=-2 \pi \int_{0}^{\infty} d r r^{2}\left[e^{-\beta u_{\mathrm{pp}}(r)}-1\right] .
$$

If we define

$$
\Delta B=B_{\mathrm{mm}}-B_{\mathrm{pp}}=2 \pi \int_{0}^{\infty} d r r^{2}\left[e^{-\beta U(r)}-1\right],
$$

which is minus the second virial coefficient for the effective pp interaction $U$, then we find up to a constant, using the Flory approach of Ref. [18] outlined above,

$$
\begin{aligned}
\Gamma_{\text {Flory }}[\alpha ; \Phi] \equiv & \frac{3}{2}\left(\alpha^{-2}+\alpha^{2}\right)+\frac{V_{1} N^{1 / 2}}{\alpha^{3}}+\frac{V_{2}^{2}}{2 \alpha^{6}} \\
& +N[\Phi \ln \Phi+(1-\Phi) \ln (1-\Phi)] \\
& -\frac{V_{1} N^{1 / 2}}{\alpha^{3}} \frac{\Delta B}{B_{\mathrm{mm}}} \Phi^{2},
\end{aligned}
$$

where $V_{1}=B_{\mathrm{mm}} / b^{3}, V_{2}=C^{1 / 2} / b^{3}$, and $\Phi$ is the average occupation $(C>0$ is the third virial coefficient). To follow Ref. [18], we have also replaced $-2 \ln \alpha$ in $\Gamma$ (A9) by $\frac{3}{2} \alpha^{-2}$ to (heuristically) recover the correct polymer swelling, $\alpha=1$, in the absence of $\mathrm{mm}$ and $\mathrm{pp}$ interactions (we could have as easily, following de Gennes [32], replaced $-2 \ln \alpha$ by $-3 \ln \alpha$ ). The above expression for $\Gamma_{\text {Flory }}$ is in agreement with the Flory approach of Ref. [18].

In the absence of bound particles, the polymer is assumed to be in a swollen state $\left(V_{1}>0\right)$. The first line in (A17) is the usual Flory expression for a bare polymer. The second line is the usual entropy of mixing for a noninteracting lattice gas and the third line arises from the coupling between the bound particles and the fluctuating polymer.

By following Ref. [18] and replacing $b^{3} P_{i j}(r ; R)$ by an effective interaction volume $R^{-3}=\left(\alpha R_{\mathrm{G}}\right)^{-3}$, describing (approximatively) the volume occupied by the polymer, we have lost all notion of the range of the effective 1D pp interaction along the polymer. We also recall that applying mean field theory in such a situation can be extremely misleading, because mean field theory will always lead to a phase transition, even for the 1D SRLG for which no phase transition exists.

It appears at first sight from (A17) that phase separation can take place if the pp interaction is less repulsive (but not necessarily attractive) than the $\mathrm{mm}$ one, i.e., $\Delta B>0$. Below we will show that a sufficiently strong attractive pp interaction is actually necessary to obtain phase separation because the
Flory approach requires concomitant polymer collapse (to a globule state). The parameter $k$ introduced in Ref. [18] is related to the second virial coefficients introduced above by

$$
k \equiv \frac{\Delta B}{B_{\mathrm{mm}}}-1 .
$$

The system of equations governing the equilibrium behavior of the coupled system can be derived from (A17) using the Flory minimization condition and the definitions of the normalized chemical potential $\tilde{\mu}_{\text {Flory }}=\beta \mu_{\text {Flory }}$ and pressure $\tilde{P}_{\text {Flory }}=\beta P_{\text {Flory }}$,

$$
\begin{aligned}
\left(\partial \Gamma_{\text {Flory }} / \partial \alpha\right)_{\Phi} & =0, \\
N^{-1}\left(\partial \Gamma_{\text {Flory }} / \partial \Phi\right)_{N} & =\tilde{\mu}_{\text {Flory }}, \\
-\left(\partial \Gamma_{\text {Flory }} / \partial N\right)_{M} & =\tilde{P}_{\text {Flory }},
\end{aligned}
$$

where $M=N \Phi$ (the average number of particles on the polymer), which leads to

$$
\begin{aligned}
\alpha^{5}-\alpha & =V_{1} N^{1 / 2}\left[1-(1+k) \Phi^{2}\right]+\frac{V_{2}^{2}}{\alpha^{3}}, \\
\tilde{\mu}_{\text {Flory }} & =-\frac{2 V_{1}(1+k) \Phi}{\alpha^{3} N^{1 / 2}}+\ln \left(\frac{\Phi}{1-\Phi}\right), \\
\tilde{P}_{\text {Flory }} & =-\frac{V_{1}\left[1+3(1+k) \Phi^{2}\right]}{2 \alpha^{3} N^{1 / 2}}-\ln (1-\Phi) .
\end{aligned}
$$

Dormidontova et al. [18] used the system of equations (A20) to study phase behavior [see their Eq. (5.3)] numerically for a large but finite value of $N$ (equal to $10^{4}$ ). They found phase separation only in the globule state. They therefore concluded that bound-particle phase separation was necessarily linked to the collapse of the polymer with particle occupation on both branches of the phase coexistence curve necessarily very high $(>0.75)$.

We can better understand the results of Ref. [18] and facilitate the comparison with our own results by working in the thermodynamic limit $(N \rightarrow \infty)$, where the system of equations (A20) can be simplified and the polymer degrees of freedom can be eliminated, leading to an effective theory for the bound particles. As shown in Figs. 5 and 6 of Ref. [18], phase diagrams at room temperature can conveniently be presented in the $(\Phi, k)$ plane, where $k>0$ parametrizes the amplitude of the attractive pp interaction with respect to the amplitude of the repulsive $\mathrm{mm}$ one. The dividing $(\theta)$ curve in the $(\Phi, k)$ plane between the swollen state (to the left) and the globule state (to the right) is determined by the vanishing of the full polymer second virial contribution, i.e. the term proportional to $V_{1}$ in the first equation of the system (A20): $k_{\theta}(\beta)=\beta^{-2}-1$. Our simple strategy is to look for phase separation first in the swollen state and then in the globule state. If we find phase separation, to be consistent, we must then check that the predicted phase diagram falls entirely in the state assumed at the outset.

In the swollen state [to the left of $k_{\theta}(\beta)$ ], we find

$$
\alpha_{\mathrm{S}}^{5} \approx V_{1} N^{1 / 2}\left[1-(1+k) \Phi^{2}\right]
$$

and therefore $\alpha_{\mathrm{S}} \propto N^{1 / 10}$, which yields the usual Flory result, $R_{\text {Flory }} \propto N^{3 / 5}$ for the end-to-end distance of a swollen polymer. The attractive pp interactions simply lead to a reduced 
effective Kuhn length. The polymer is still swollen, but less so than in the absence of bound particles. The key point now is that the pp interaction terms in the pressure and chemical potential vanish as $1 /\left(\alpha_{\mathrm{S}} N^{1 / 2}\right)=N^{-4 / 5}$, when $N \rightarrow$ $\infty$ [see (A20)] and thus the bound particle system reduces to a noninteracting lattice gas. The Flory approach severely underestimates the importance of bridging interactions in the swollen state and therefore fails to predict bound-particle phase separation, in contrast to what we found using the correct kernel (see main text).

In the globule state [to the right of $k_{\theta}(\beta)$ ], the total second virial contribution becomes negative (attractive) and polymer collapse to a compact state is arrested by the repulsive third virial contribution. In this case,

$$
\frac{V_{2}^{2}}{\alpha_{\mathrm{Gl}}^{3}} \approx V_{1} N^{1 / 2}\left[(1+k) \Phi^{2}-1\right]
$$

and therefore $\alpha_{\mathrm{Gl}} \propto N^{-1 / 6}$, which yields the usual Flory result, $R_{\mathrm{Gl}} \propto N^{1 / 3}$ for the end-to-end distance of a compact polymer (globule). The attractive pp interactions are so strong in this case that they overcome the mm repulsion. The key point now is that the pp interaction terms in the pressure and chemical potential no longer vanish when $N \rightarrow \infty$, but scale in such a way as to lead to a well-defined thermodynamic limit in the globule state:

$$
\begin{aligned}
\tilde{\mu}_{\mathrm{F}, \mathrm{Gl}}= & -2\left(\frac{V_{1}}{V_{2}}\right)^{2}(1+k) \Phi\left[(1+k) \Phi^{2}-1\right] \\
& +\ln \left(\frac{\Phi}{1-\Phi}\right), \\
\tilde{P}_{\mathrm{F}, \mathrm{Gl}}= & -\frac{1}{2}\left(\frac{V_{1}}{V_{2}}\right)^{2}\left[1+3(1+k) \Phi^{2}\right]\left[(1+k) \Phi^{2}-1\right] \\
& -\ln (1-\Phi) .
\end{aligned}
$$

Thus, the bound particle system retains attractive pp interactions in the globule state and the Flory approach predicts bound particle phase separation. We have used the effective set of equations [(A23) and (A24)], obtained by eliminating the polymer degrees of freedom from the Flory approach, to reproduce the phase diagrams obtained numerically from the full Flory approach of [18] (their Figs. 5 and 6). The critical point $\left(\Phi_{\mathrm{c}}, k_{\mathrm{c}}\right)$ in the $(\Phi, k)$ plane is defined by

$$
\frac{\partial \tilde{\mu}}{\partial \Phi}=\frac{\partial^{2} \tilde{\mu}}{\partial \Phi^{2}}=0
$$

which can even be calculated analytically (in the thermodynamic limit): For the flexible chain (Fig. 5 of Ref. [18]), $V_{1}=$ $V_{2}=1$, and we find $\left(\Phi_{\mathrm{c}}, k_{\mathrm{c}}\right)=(4 / 5,9 / 16)$ in reasonably good agreement with the critical point found in Ref. [18] for $N=10^{4}$.

We believe, however, that this phase separation in the globule state presents an inconsistency: As mentioned in the main text, in the thermodynamic limit of a globule state we expect ideal Gaussian behavior for interior monomers owing to internal screening of polymer self-avoidance. In our approach, we therefore assimilate the globule state in the thermodynamic limit to an ideal polymer with phase behavior different from that presented in Dormidontova et al. (their Figs. 5, 6, and 7). The subtlety here arises from the different scaling behavior in this case between the scaling of the endto-end distance $R$ as a function of $N$ and the scaling of $R_{i, j}$ as a function of $|i-j|$ for internal monomers (far from the surface of the polymer globule in 3D space). The correct scaling can only be obtained if the correct order of limits is taken: $N$ should be taken to infinity before $|i-j|$ to find the correct asymptotic behavior in the thermodynamic limit. Because the Flory approach therefore overestimates the overall effect of the attractive interactions between bound particles in the globule, we are not convinced that the phase behavior found by Dormidontova et al. in the globule regime has any physical reality.

\section{APPENDIX B: MEAN FIELD THEORY}

The variational method gives mean field results when only the variational chemical potential term is kept in the reference Hamiltonian $H_{0}^{\prime}$ and all correlation terms in $\Delta H^{\prime}$ :

$$
H_{0}^{\prime}=-\mu_{0}^{\prime} \sum_{i=1}^{N} \Phi_{i}
$$

and

$$
\begin{aligned}
\Delta H^{\prime}= & -J \sum_{i=1}^{N-1} \Phi_{i+1} \Phi_{i}-\frac{1}{2} \sum_{i, j}^{N} \Phi_{i} G_{i j} \Phi_{j} \\
& -\left(\mu-\mu_{0}^{\prime}\right) \sum_{i=1}^{N} \Phi_{i} .
\end{aligned}
$$

The prime on the sum means that $|i-j| \geqslant n_{\text {inf }}$, where $n_{\text {inf }}$ is the minimal internal distance in number of sites over which two particles can interact at long range. Since the correlation length vanishes in MFT, we have directly $\left\langle\Phi_{i} \Phi_{j}\right\rangle=\Phi_{0}^{\prime 2}$ for $i \neq j$, which leads to the MFT trial grand potential $\Omega_{\mathrm{V}}^{\prime}$ :

$$
\begin{aligned}
\Omega_{\mathrm{V}}^{\prime}= & -N k_{B} T \ln \left(1+e^{\beta \mu_{0}^{\prime}}\right)-N \Phi_{0}^{\prime 2}(J+S) \\
& -N\left(\mu-\mu_{0}^{\prime}\right) \Phi_{0}^{\prime}
\end{aligned}
$$

with

$$
\Phi_{0}^{\prime}=\frac{e^{\beta \mu_{0}^{\prime}}}{1+e^{\beta \mu_{0}^{\prime}}} .
$$

The optimization equation, $\partial \Omega_{\mathrm{V}}^{\prime} / \partial \mu_{0}^{\prime}=0$, has as solution $\mu_{0}^{\prime \star}$, which gives directly

$$
\mu=\mu_{0}^{\prime \star}-2 \Phi_{0}^{\prime \star}(J+S)
$$

and $\mu_{0}^{\prime \star}$ as a function of $\Phi_{0}^{\prime \star}$ is obtained by inverting Eq. (B4):

$$
\beta \mu_{0}^{\prime \star}=\ln \left(\frac{\Phi_{0}^{\prime \star}}{1-\Phi_{0}^{\prime \star}}\right) \text {. }
$$

The definition of MFT mean occupancy, $\Phi$, together with the optimization condition, leads to $\Phi=\Phi_{0}^{\prime \star}$. The MFT LRLG result for the grand potential is then

$$
\frac{\Omega_{\mathrm{V}}^{\prime \star}}{N}=k_{B} T \ln (1-\Phi)+\Phi^{2}(J+S) .
$$

The MFT result for the chemical potential is given by

$$
\mu_{\mathrm{MF}}=k_{B} T \ln \left(\frac{\Phi}{1-\Phi}\right)-2 \Phi(J+S)
$$




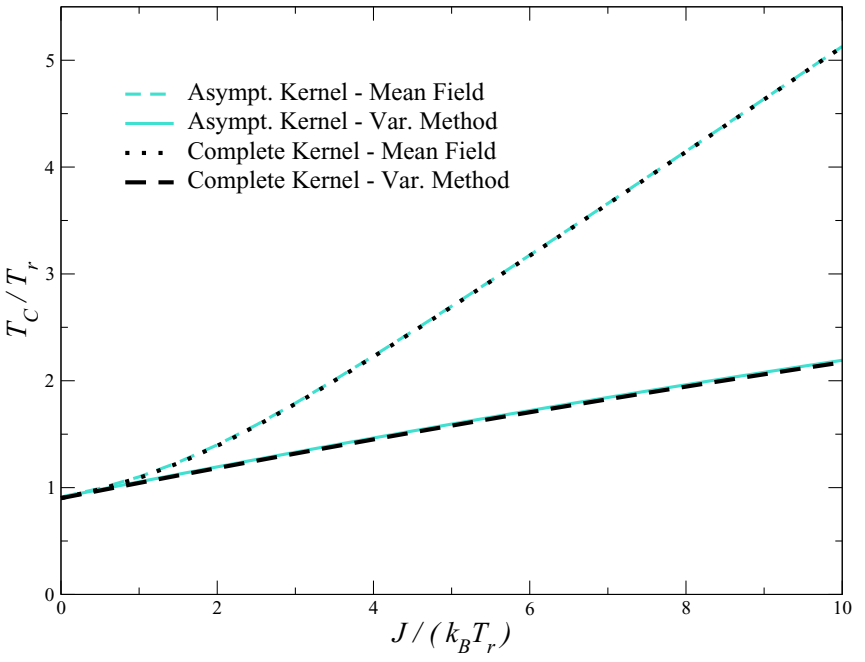

FIG. 4. Critical temperature (normalized by $T_{r}=300 \mathrm{~K}$ ) as function of $J / k_{B} T_{r}$ using the Gaussian complete (black) and asymptotic kernel (turquoise). Solid (dotted) line is for variational (mean field) method.

and the MFT pressure by

$$
P_{\mathrm{MF}}=-\Omega_{\mathrm{V}}^{\prime \star} /\left(l_{m} N\right) .
$$

In MFT, the long-range interaction simply additively renormalizes the NN interaction via $J \rightarrow J+S$ with the additional complication that $S$ is temperature dependent.

\section{APPENDIX C: DEFINITION OF $S$ AND OTHERS SUMS}

For the ParABS system, the large value of the DNA persistence length leads to a relatively large value $\left(n_{\text {inf }}=10\right)$ for the lower cutoff, which allows us to use the asymptotic large- $k$ form of the kernel, written as

$$
K_{k}=K_{\mathrm{SW}} k^{-\alpha},
$$

in evaluating the sums $S, S^{\prime}$ and $S^{\prime \prime}$. This approximation simplifies the numerical calculations necessary to obtain the phase diagram.

Because the complete Gaussian polymer probability distribution function (PDF) takes a simple form [33,43],

$$
P_{i j}^{\mathrm{G}}(R)=\left(\frac{3}{2 \pi\left[R_{i j}^{\mathrm{G}}\right]^{2}}\right)^{3 / 2} \exp \left[-\frac{3}{2}\left(\frac{R}{R_{i j}^{\mathrm{G}}}\right)^{2}\right]
$$

(where $R_{i j}^{\mathrm{G}}=b|i-j|^{1 / 2}$ with $b$ the Kuhn length), we were able to calculate the complete kernel analytically for this case.

Using this complete kernel, we then validated the asymptotic approximation by comparing the phase diagrams obtained with those obtained using the complete Gaussian kernel. In Fig. 4, we show that the critical temperatures predicted by the Gaussian complete and asymptotic kernel behave similarly. We assume that the same positive conclusion concerning the validity of this asymptotic approximation can be drawn for the self-avoiding polymer (SAP), for which the complete PDF and a fortiori the kernel are not known analytically. Hence, for calculational efficiency in the thermodynamic limit, we chose to rewrite $S$ as

$$
\begin{aligned}
S & \approx \sum_{k=n_{\text {inf }}}^{\infty} K_{k}=\sum_{k=1}^{\infty} K_{k}-\sum_{k=1}^{n_{\text {inf }}-1} K_{k} \\
& =K_{\text {SW }}\left[\zeta(\alpha)-\sum_{k=1}^{n_{\text {inf }}-1} k^{-\alpha}\right],
\end{aligned}
$$

where $\zeta(x)$ is the Riemann zeta function. The first term $K_{\mathrm{SW}} \zeta(\alpha)$ is the complete asymptotic contribution to the sum $S$. The second term allows us to incorporate the influence of the polymer persistence length.

The same procedure can be applied to the sums $S^{\prime}$ and $S^{\prime \prime}$, leading to

$$
S^{\prime}=K_{\mathrm{SW}}\left[\mathrm{Li}_{\alpha}\left(r_{\mathrm{LG}}\right)-\sum_{k=1}^{n_{\mathrm{inf}}-1} \frac{r_{\mathrm{LG}}^{k}}{k^{\alpha}}\right]
$$

and

$$
S^{\prime \prime}=K_{\mathrm{SW}}\left[\mathrm{Li}_{\alpha-1}\left(r_{\mathrm{LG}}\right)-\sum_{k=1}^{n_{\mathrm{inf}}-1} \frac{r_{\mathrm{LG}}^{k}}{k^{\alpha-1}} .\right],
$$

where

$$
\operatorname{Li}_{s}(z) \equiv \sum_{k=1}^{\infty} \frac{z^{k}}{k^{s}}
$$

is the polylogarithm function.

The same decomposition carried out for the complete kernel $G_{i j}$ leads to

$$
\begin{aligned}
S & =\sum_{k=n_{\text {inf }}}^{\infty} G_{k}=\sum_{k=1}^{\infty} G_{k}-\sum_{k=1}^{n_{\text {inf }}} G_{k} \\
& \approx K_{\mathrm{SW}} \zeta(\alpha)+\sum_{k=1}^{n_{\text {sup }}}\left(G_{k}-K_{\mathrm{SW}} k^{-\alpha}\right)-\sum_{k=1}^{n_{\text {inf }}-1} G_{k} .
\end{aligned}
$$

The first and last terms have been previously explained. The second term takes into account the residual difference between the complete kernel $G_{k}$ and its asymptotic form, which is most important for low values of $k$. These two forms for the kernel converge very quickly and for practical purposes we take $n_{\text {sup }}=50$. The same transformation can be applied to the sums $S^{\prime}$ and $S^{\prime \prime}$, leading to

$$
\begin{aligned}
S^{\prime}= & \sum_{k=n_{\text {inf }}}^{\infty} G_{k} r_{\mathrm{LG}}^{k} \\
\approx & K_{\mathrm{SW}} \operatorname{Li}_{\alpha}\left(r_{\mathrm{LG}}\right)+\sum_{k=1}^{n_{\text {sup }}}\left(G_{k}-K_{\mathrm{SW}} k^{-\alpha}\right) r_{\mathrm{LG}}^{k} \\
& -\sum_{k=1}^{n_{\text {inf }}-1} G_{k} r_{\mathrm{LG}}^{k}
\end{aligned}
$$


and

$$
\begin{aligned}
S^{\prime \prime}= & \sum_{k=n_{\text {inf }}}^{\infty} G_{k} k r_{\mathrm{LG}}^{k} \\
\approx & K_{\mathrm{SW}} \operatorname{Li}_{\alpha-1}\left(r_{\mathrm{LG}}\right)+\sum_{k=1}^{n_{\text {sup }}}\left(G_{k}-K_{\mathrm{SW}} k^{-\alpha}\right) k r_{\mathrm{LG}}^{k} \\
& -\sum_{k=1}^{n_{\text {inf }}-1} G_{k} k r_{\mathrm{LG}}^{k} .
\end{aligned}
$$

\section{APPENDIX D: VARIATIONAL AND MEAN-FIELD CRITICAL TEMPERATURE}

The self-consistent equation for the variational critical temperature $T_{\mathrm{c}}^{\mathrm{V}}$ can be found using the variational expression for the chemical potential $\mu$ :

$$
\begin{aligned}
\mu-\mu_{0}^{\star}= & 2 \Phi_{0}^{\star}\left[S^{\prime}-S\right]-S^{\prime} \\
& -\Phi_{0}^{\star}\left(1-\Phi_{0}^{\star}\right)\left(1-2 \Phi_{0}^{\star}\right) S^{\prime \prime} f(\Phi),
\end{aligned}
$$

with the optimized variational parameter $\mu_{0}^{\star}$ given in Eq. (21) and the function

$$
f(\Phi)=\beta\left(\frac{\partial \Phi_{0}}{\partial \mu_{0}}\right)_{\mu_{0}=\mu_{0}^{\star}}^{-1}=\frac{4\left[\sinh ^{2}(Y(\Phi))+B\right]^{\frac{3}{2}}}{B \cosh (Y(\Phi))} .
$$

The objective is to find first the equation for the coexistence temperature as a function of $\Phi$ using the equality of the chemical potential in the low and high occupation states (along with hole-particle symmetry)

$$
\mu\left(\Phi_{v}\right)=\mu\left(1-\Phi_{v}\right)
$$

and then to develop this expression for $\Phi \rightarrow \Phi_{\mathrm{c}}=1 / 2$ (or $A \rightarrow 0$ ) to find the critical temperature. The first step yields an implicit equation for the variational prediction for the coexistence curve:

$$
\begin{aligned}
2 k_{B} T & \ln \left(\frac{\sqrt{1-A_{v}^{2}+B A_{v}^{2}}+A_{v} \sqrt{B}}{\sqrt{1-A_{v}^{2}+B A_{v}^{2}}-A_{v} \sqrt{B}}\right) \\
& -2 \Phi_{v}\left(S-S_{v}^{\prime}\right)+2\left(1-\Phi_{v}\right)\left(S-S_{l}^{\prime}\right) \\
& +\Phi_{v}\left(1-\Phi_{v}\right) A_{v}\left[S_{v}^{\prime \prime} f\left(\Phi_{v}\right)+S_{l}^{\prime \prime} f\left(1-\Phi_{v}\right)\right]=0,
\end{aligned}
$$

with $A_{v}=2 \Phi_{v}-1, S_{v}^{\prime}=S^{\prime}\left(\Phi_{v}\right)$, and $S_{l}^{\prime}=S^{\prime}\left(\Phi_{l}\right)$. To carry out the second step and find $T_{\mathrm{c}}^{\mathrm{V}}$, we take the limit $\Phi \rightarrow 1 / 2$. One can easily show that in this limit $S_{v}^{\prime}, S_{l}^{\prime} \rightarrow S^{\prime}$ because $r_{L G} \rightarrow(1-\sqrt{B}) /(1+\sqrt{B})$. The same statement holds for $S_{v}^{\prime \prime}, S_{l}^{\prime \prime} \rightarrow S^{\prime \prime}$. Moreover, $f(\Phi) \rightarrow 4 \sqrt{B}$ and equation (D4) becomes

$$
2 k_{B} \sqrt{B_{\mathrm{c}}} T_{\mathrm{c}}^{\mathrm{V}}-\left(S-S^{\prime}\right)+S^{\prime \prime} \sqrt{B_{\mathrm{c}}}=0,
$$

with $B_{\mathrm{c}}=\exp \left[-J /\left(k_{B} T_{\mathrm{c}}\right)\right]$, and finally we obtain an implicit equation for $T_{\mathrm{c}}^{\mathrm{V}}$ :

$$
T_{\mathrm{c}}^{\mathrm{V}}=\frac{S_{\mathrm{c}}-S_{\mathrm{c}}^{\prime}}{2 k_{B} \sqrt{B_{\mathrm{c}}}}-\frac{S_{\mathrm{c}}^{\prime \prime}}{2 k_{B}}
$$

with $B_{\mathrm{c}}, S_{\mathrm{c}}, S_{\mathrm{c}}^{\prime}$, and $S_{\mathrm{c}}^{\prime \prime}$ evaluated at the critical point (and therefore functions of $T_{\mathrm{c}}^{\mathrm{V}}$ ).

Developing the last expression for $J \rightarrow 0$ and using the result that $S^{\prime}, S^{\prime \prime} \rightarrow 0$ in this limit lead to the self-consistent

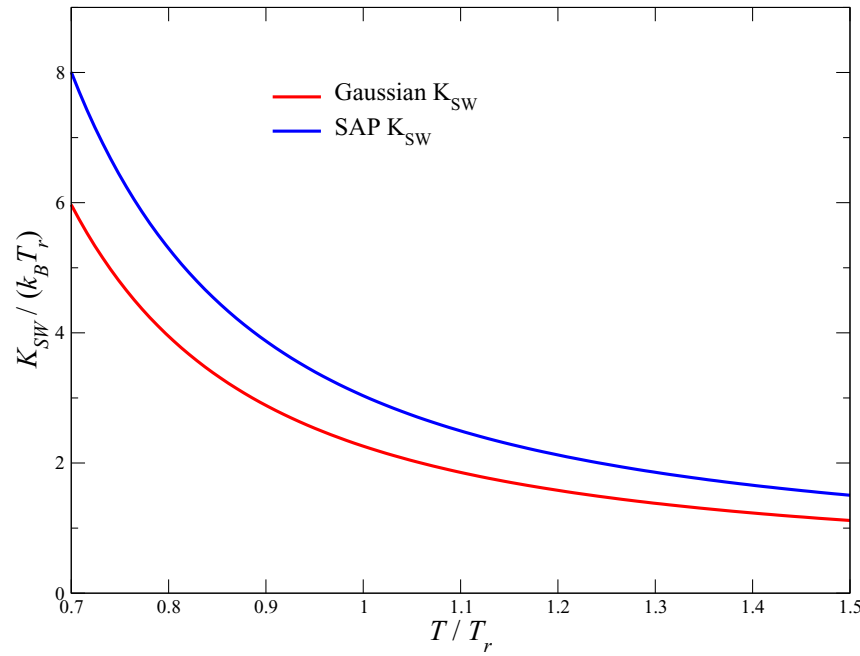

FIG. 5. Kernel intensity $K_{\mathrm{SW}} /\left(k_{B} T_{r}\right)$ as a function of $T / T_{r}$. Blue (red) is for the SAP (Gaussian) statistics.

equation for the mean field critical temperature:

$$
\frac{T_{\mathrm{c}}^{\mathrm{MFT}}}{T_{\mathrm{r}}}=\frac{1}{2 k_{\mathrm{B}} T_{\mathrm{c}}^{\mathrm{MFT}}}\left[J+S\left(T_{\mathrm{c}}^{\mathrm{MFT}}\right)\right],
$$

with $S(T)$ given by Eq. (C3) in the asymptotic approximation to the kernel.

Because of the temperature dependence of the kernel, the MFT critical temperature $T_{\mathrm{c}}^{\mathrm{MFT}}$, which can also be obtained directly from

$$
\left(\partial P_{\mathrm{MF}} / \partial \Phi\right)_{\Phi=\Phi_{\mathrm{c}}}=0,
$$

is also a solution to an implicit equation. If $T_{\mathrm{c}}^{\mathrm{MFT}} / T_{\mathrm{r}}>1$, then the MFT critical temperature can be estimated by replacing $T_{\mathrm{c}}^{\mathrm{MFT}}$ with $T_{\mathrm{r}}$ in Eq. (D7) to obtain an explicit analytical approximation evoked in the main text:

$$
\frac{T_{\mathrm{c}}^{\mathrm{MFT}}}{T_{\mathrm{r}}} \approx \frac{1}{2 k_{\mathrm{B}} T_{\mathrm{r}}}\left[J+S\left(T_{\mathrm{r}}\right)\right]
$$

This approximation relies on the relatively weak temperature dependence of $K_{\mathrm{SW}}$ for the chosen ParBS model parameters when $T>T_{\text {r }}$ (see Fig. 5).

\section{APPENDIX E: GENERALIZATION TO MODELS (I) AND (II) OF THE INTRODUCTION}

For model (i), the bound particles are not fluctuating but rather quenched, and the problem reduces to a heterogeneous polymer problem where the sequence of occupied sites is frozen. The key question to address is how this quenched particle occupation influences polymer statistics, including polymer collapse at sufficiently high frozen particle density $[21,22,27,28]$. In certain cases, we expect to find a strong coupling between the sequence and the polymer conformation with possible applications in the area of intrinsically disordered proteins (IDPs) (see, e.g., Ref. [57]).

For model (ii), nonattracting mobile bound particles fluctuate and can bind simultaneously to two (or more, in some cases) polymer sites to form bridges [19,23,29]. This model 
can be formulated by modifying the present model: The nearest-neighbor interaction should be dropped and the longrange $3 \mathrm{D}$ spatial interaction should written as $\Phi_{i}\left(1-\Phi_{j}\right)=$ $-\Phi_{i} \Phi_{j}+\Phi_{i}$, instead of $\Phi_{i} \Phi_{j}$, to take into account that an occupied site $i$ can interact attractively with an unoccupied site $j$ (a hole). The sign of the long-range interaction is changed with respect to model (iii), becoming repulsive. Although we probably would not expect a true phase transition in this case, a more detailed study needs to be performed before drawing any solid conclusions. We would, however, expect a substantial modification of loop entropy and polymers statistics, leading to possible polymer collapse, depending on the average particle occupancy on the polymer (as already observed in simulations).
[1] R. Horwitz, Cellular biophysics, Biophys. J. 110, 993 (2016).

[2] A. A. Hyman, C. A. Weber, and F. Jülicher, Liquid-liquid phase separation in biology, Annu. Rev. Cell Dev. Biol. 30, 39 (2014).

[3] C. P. Brangwynne, Phase transitions and size scaling of membrane-less organelles, J. Cell Biol. 203, 875 (2013).

[4] C. P. Brangwynne, P. Tompa, and R. V. Pappu, Polymer physics of intracelullar phase transitions, Nat. Phys. 11, 899 (2015).

[5] C. F. Lee, C. P. Brangwynne, J. Gharakhani, A. A. Hyman, and F. Jülicher, Spatial Organization of the Cell Cytoplasm by Position-Dependent Phase Separation, Phys. Rev. Lett. 111, 088101 (2013).

[6] M. Feric, N. Vaidya, T. S. Harmon, D. M. Mitrea, L. Zhu, T. M. Richardson, R. W. Kriwacki, R. V. Pappu, and C. P. Brangwynne, Coexisting liquid phases underlie nucleolar subcompartments, Cell 165, 1686 (2016).

[7] A. G. Larson, D. Elnatan, M. M. Keenen, M. J. Trnka, J. B. Johnston, A. L. Burlingame, D. A. Agard, S. Redding, and G. J. Narlikar, Liquid droplet formation by HP1 $\alpha$ suggests a role for phase separation in heterochromatin, Nature (London) 547, 236 (2017).

[8] D. Schumacher, S. Bergeler, A. Harms, J. Vonck, S. HunekeVogt, E. Frey, and L. Søgaard-Andersen, The PomXYZ proteins self-organize on the bacterial nucleoid to stimulate cell division, Development. Cell 41, 299 (2017).

[9] J. F. Marko, Biophysics of protein-DNA interactions and chromosome organization, Physica A (Amsterdam, Neth.) 418, 126 (2015).

[10] N. L. Sullivan, K. A. Marquis, and D. Z. Rudner, Recruitment of SMC by ParB-parS organizes the origin region and promotes efficient chromosome segregation, Cell 137, 697 (2009).

[11] A. Sanchez, D. I. Cattoni, J.-C. Walter, J. Rech, A. Parmeggiani, M. Nollman, and J.-Y. Bouet, Stochastic self-assembly of ParB proteins builds the bacterial DNA segregation apparatus, Cell Syst. 1, 163 (2015).

[12] C. P. Broedersz, X. Wang, Y. Meir, J. J. Loparo, D. Z. Rudner, and N. S. Wingreen, Condensation and localization of the partitioning protein ParB on the bacterial chromosome, Proc. Nat. Acad. Sci. USA 111, 8809 (2014).

[13] J.-C. Walter, J. Dorignac, V. Lorman, J. Rech, J.-Y. Bouet, M. Nollman, J. Palmeri, A. Parmeggiani, and F. Geniet, Surfing on Protein Waves: Proteophoresis as a Mechanism for Bacterial Genome Partitioning, Phys. Rev. Lett. 119, 028101 (2017).

[14] A. Le Gall, D. I. Cattoni, B. Guilhas, C. Mathieu-Demaziere, L. Oudjedi, J. B. Fiche, J. Rech, S. Abrahamsson, H. Murray, J. Y. Bouet, and N. Nollmann, Bacterial partition complexes segregate within the volume of the nucleoid, Nat. Commun. 7, 12107 (2016).
[15] B. Guilhas, J.-C. Walter, J. Rech, G. David, N.-O. Walliser, J. Palmeri, C. Mathieu-Demaziere, A. Parmeggiani, J.-Y. Bouet, A. Le Gall, and M. Nollmann, ATP-driven separation of liquid phase condensates in bacteria Molecular Cell 79, 293 (2020).

[16] H. Diamant and D. Andelman, Self-assembly in mixtures of polymers and small associating molecules, Macromolecules 33, 8050 (2000).

[17] A. Y. Grosberg, Collapse and intramolecular phase layering in a polymer in which each unit may be in two states, Biophysics 29, 621 (1984).

[18] E. E. Dormidontova, A. Y. Grosberg, and A. R. Khokhlov, Intramolecular phase separation in heteropolymer chain with annealed primary structure, Makromol. Chem., Theory Simul. 1, 375 (1992).

[19] J. Johnson, C. A. Brackley, P. R. Cook, and D. Marenduzzo, A simple model for DNA bridging proteins and bacterial or human genomes: Bridging-induced attraction and genome compaction, J. Phys.: Condens. Matter 27, 064119 (2015).

[20] J.-C. Walter, N.-O. Walliser, G. David, J. Dorignac, F. Geniet, J. Palmeri, A. Parmeggiani, N. S. Wingreen, and C. P. Broedersz, Looping and clustering model for the organization of proteinDNA complexes on the bacterial genome, New J. Phys. 20, 035002 (2018).

[21] V. F. Scolari and M. Cosentino Lagomarsino, Combined collapse by bridging and self-adhesion in a prototypical polymer model inspired by the bacterial nucleoid, Soft Matter 11, 1677 (2015).

[22] I. Junier, O. Martin, and F. Képès, Spatial and topological organization of DNA chains induced by gene co-localization, PLoS Comput. Biol. 6, e1000678 (2010).

[23] M. Barbieri, M. Chotalia, J. Fraser, L.-M. Lavitas, J. Dostie, A Pombo, and M. Nicodemi, Complexity of chromatin folding is captured by the strings and binders switch model, Proc. Nat. Acad. Sci. USA 109, 16173 (2012).

[24] D. Jost, P. Carrivain, G. Cavalli, and C. Vaillant. Modeling epigenome folding: Formation and dynamics of topologically associated chromatin domains, Nucl. Acids Res. 42, 9553 (2014).

[25] N. Haddad, D. Jost, and C. Vaillant, Perspectives: Using polymer modeling to understand the formation and function of nuclear compartments, Chromosome Res. 25, 35 (2017).

[26] D. Jost, C. Vaillant, and P. Meister, Coupling 1D modifications and 3D nuclear organization: Data, models, and function, Curr. Opin. Cell Biol. 44, 20 (2017).

[27] J. D. Bryngelson and D. Thirumalai, Internal Constraints Induce Localization in an Isolated Polymer Molecule, Phys. Rev. Lett. 76, 542 (1996). 
[28] Y. Kantor and M. Kardar, Conformations of randomly linked polymers, Phys. Rev. E 54, 5263 (1996).

[29] G. Le Treut, F. Képès, and H. Orland, Phase behavior of DNA in the presence of DNA-binding proteins, Biophys J. 110, 51 (2016).

[30] J. E. Mayer and M. G. Mayer, Statistical Mechanics, 2nd ed. (John Wiley \& Sons, New York, 1977).

[31] R. P. Feynman, Statistical Mechanics: A Set of Lectures (W. A. Benjamin, Reading, MA, 1972).

[32] P. De Gennes, Collapse of a polymer chain in poor solvents, J. Phys. Lett. 36, 55 (1975).

[33] P.-G. de Gennes, Scaling Concepts in Polymer Physics (Cornell University Press, Ithaca, NY, 1979).

[34] P. Flory, Principles of Polymer Chemistry (Cornell University Press, Ithaca, NY, 1971).

[35] A. Y. Grosberg and A. R. Khokhlov, Statistical Physics of Macromolecules (AIP Press, New York, 1994).

[36] G. Fredrickson, The Equilibrium Theory of Inhomogeneous Polymers (Clarendon Press, Oxford, UK, 2006).

[37] E. Luijten and H. W. J. Blöte, Classical critical behavior of spin models with long-range interactions, Phys. Rev. B 56, 8945 (1997).

[38] P. W. Anderson and G. Yuval, Some numerical results on the Kondo problem and the inverse square one-dimensional Ising model, J. Phys. C 4, 607 (1971).

[39] F. J. Dyson, Existence of a phase-transition in a onedimensional Ising ferromagnet, Commun. Math. Phys. 12, 91 (1969).

[40] D. Mukamel, Notes on the statistical mechanics of systems with long-range interactions, arXiv:0905.1457 [cond-mat.statmech].

[41] J. M. Kosterlitz and D. J. Thouless, Ordering, metastability and phase transitions in two-dimensional systems, J. Phys. C: Solid State Phys. 6, 1181 (1973).

[42] R. Lua, R. L. Borovinskiy, and A. Y. Grosberg, Fractal and statistical properties of large compact polymers: A computational study, Polymer 45, 717 (2004).

[43] H. Schiessel, Biophysics for Beginners: A Journey through the Cell Nucleus (Pan Stanford Publishing, Singapore, 2014).

[44] K. G. Petrosyan and C.-K. Hu, Protein-mediated loops and phase transition in nonthermal denaturation of DNA, J. Stat. Mech. (2011) P01005.
[45] L. J. Kijewski and M. P. Kawatha, One-dimensional Ising model with long-range interaction, Phys. Lett. A 31, 479 (1970).

[46] B. McCoy and T. T. Wu, The Two-Dimensional Ising Model (Harvard University Press, Cambridge, UK, 1973).

[47] K. Huang, Statistical Physics, 2nd ed. (John Wiley, New York, 1987).

[48] L. D. Landau and E. M. Lifshitz, Statistical Physics, 3rd ed., Course of Theoretical Physics, Vol. 5 (Pergamon Press, Oxford, UK, 1980).

[49] R. E. Debaugny, A. Sanchez, J. Rech, D. Labourdette, J. Dorignac, F. Geniet, J. Palmeri, A. Parmeggiani, F. Boudsocq, V. A. Leberre, J.-C. Walter, and J.-Y. Bouet, Conserved mechanism for dynamic partition complexes assembly on bacterial chromosomes and plasmids, Mol. Syst. Biol. 14, e8516 (2018).

[50] J.-Y. Bouet, M. Stouf, E. Lebailly, and F. Cornet, Mechanisms for chromosome segregation, Curr. Opin. Microbiol. 22, 60 (2014).

[51] O. Rodionov, M. Lobocka, and M. Yarmolinsky, Silencing of genes flanking the P1 plasmid centromere, Science 283, 546 (1999).

[52] J.-Y. Bouet, J. Rech, S. Egloff, D. P. Biek, and D. Lane, Probing plasmid partition centromere-based incompatibility, Mol. Microbiol. 55, 511 (2005).

[53] R. Everaers, S. Kumar, and C. Simm, Unified description of poly- and oligonucleotide DNA melting: Nearest-neighbor, Poland-Sheraga, and lattice models, Phys. Rev. E 75, 041918 (2007).

[54] In contrast, a Flory approach [18] does not lead to phase separation in the thermodynamic limit for Gaussian and self-avoiding polymers (see Appendix A).

[55] T. G. W. Graham, X. Wang, D. Song, C. M. Etson, A. M. van Oijen, D. Z. Rudner, and J. J. Loparo, ParB spreading requires DNA bridging, Genes Dev. 28, 1228 (2014).

[56] G. L. M. Fisher, C. L. Pastrana, V. A. Higman, A. Koh, J. A. Taylor, A. Butterer, T. Craggs, F. Sobott, H. Murray, M. P. Crump, F. Moreno-Herrero, and M. S. Dillingham, The structural basis for dyanmic DNA binding and bridging interactions which condense the bacterial centromere, eLife 6, e28086 (2017).

[57] V. N. Uversky, Intrinsically disordered proteins and their mysterious (meta)physics, Front. Phys. 7, 10 (2019). 